\title{
NEURONAL DEATH IN THE SPINAL GANGLIA OF THE CHICK EMBRYO AND ITS REDUCTION BY NERVE GROWTH FACTOR ${ }^{1}$
}

\author{
V. HAMBURGER, J. K. BRUNSO-BECHTOLD, ${ }^{2}$ AND J. W. YIP ${ }^{3}$ \\ Department of Biology, Washington University, St. Louis, Missouri 63130
}

\begin{abstract}
In the spinal ganglia of the chick embryo, two neuronal populations can be distinguished: large, early differentiating ventrolateral (VL) cells and small, late differentiating dorsomedial (DM) cells. It was found that, beginning with stage 25 , the DM cells originate from a narrow band of small, immature cells at the medial border of the ganglion, extending to the dorsolateral border. We have designated this band as the inner and outer marginal zone.

Neuronal death was investigated in thoracic ganglion 18 and brachial ganglion 15 by counting degenerating cells, separately for the VL and DM populations, at every stage from stage 24 (41/2 days) to stage 38 (12 days). In both ganglia, separate degeneration periods were found for the VL and DM populations which do not overlap. The peaks of degeneration are: stage 27 ( $51 / 2$ days) for the VL population in ganglion 18, stage 30 (6 $6^{1 / 2}$ to 7 days) for VL in ganglion 16 , and stage 35 ( $8 \frac{1}{2}$ days) for DM in both ganglia.

Daily injections of $6 \mu \mathrm{g}$ of nerve growth factor, (NGF) in 6 to $12 \mu \mathrm{l}$ of $0.9 \%$ sterile salt solution into the yolk sac from stage 21 ( $3 \frac{1 / 2}{2}$ days) to the day of sacrifice resulted in a significant reduction of neuronal death in the VL population of ganglion 18 and in the rescue of practically all VL neurons in ganglion 15 and all DM neurons in both ganglia, which normally would have died. This is the first demonstration of an NGF effect on VL neurons.
\end{abstract}

In the analysis of the multiple effects of nerve growth factor (NGF), the sympathetic ganglia have played the dominant role; the sensory ganglia which are the other target of NGF have attracted much less attention. Yet, if one entertains the notion that NGF might be the natural trophic maintenance agent for these two neuron types (i.e., that NGF is actually produced by their target organs), then one approach to the testing of this hypothesis would be to engage in in vivo experiments. For this

\footnotetext{
${ }^{1}$ Dedicated to Dr. Rita Levi-Montalcini.

This work was supported by Grant NS05721 from the National Institute of Neurological Communicative Disorders and Stroke, National Institutes of Health and a grant from the Jerry Lewis Neuromuscular Research Center to Washington University School of Medicine. We are grateful to Drs. Bradshaw, Costrini, and Ruben of the Department of Biochemistry, Washington University School of Medicine and Dr. Shooter of the Department of Neurobiology, Stanford University School of Medicine for the generous supply of nerve growth factor. We thank Mr. Cramer Lewis of the Department of Medical Illustrations, Washington University School of Medicine for the photographic work and Ms. Vicki Friedman of the Department of Surgical Illustrations for execution of the line drawings. We also thank Mrs. Helen Gregory for very competent technical assistance and Mrs. Irma Morose for typing the manuscript.

${ }^{2}$ Present address: Department of Anatomy, Vanderbilt University School of Medicine, Nashville, TN 37215.

${ }^{3}$ Present address: Department of Physiology, University of Pittsburgh School of Medicine, Pittsburgh, PA 15261.
}

undertaking, sensory ganglia would be preferable to sympathetic ganglia, because their early differentiation processes, including cell loss by degeneration, are much better known than those of sympathetic ganglia.

Since a direct attack on this problem, for instance an attempt to isolate NGF from specific embryonic tissues seems unrealistic at this time, one has to resort to the marshaling of indirect evidence. The first step was the demonstration that NGF is transported retrogradely and selectively from the hindlimb to lumbar dorsal root ganglia of chick embryos at a stage ( $10 \mathrm{~d}$ of incubation) when the ganglia are known to be responsive to NGF (BrunsoBechtold and Hamburger, 1979). The next step is to test the role of NGF as a trophic maintenance agent in vivo. We know that NGF is essential for the survival of dissociated sensory and sympathetic neurons in vitro (LeviMontalcini and Angeletti, 1963; Green, 1977). We have now addressed the question of whether NGF can alleviate normally occurring neuronal death in sensory ganglia in the embryo.

In a previous study (Hamburger and Levi-Montalcini, 1949), distinct patterns of distribution of degenerating cells in spinal ganglia had been recognized. First, neuronal death was found to be much more extensive in cervical and thoracic ganglia than in limb-innervating ganglia. This differential accounts, in part, for the conspicuous volume differences between the two groups of ganglia. In addition to this regional pattern, a temporal 
pattern was observed; massive degeneration occurs in spinal ganglia during the period between $4 \frac{1}{2}$ and $7 \frac{1}{2} \mathrm{~d}$ of incubation. Finally, there are two populations of neurons in the spinal ganglia of chick embryos; early differentiating large neurons in a ventrolateral position (VI) and smaller, late differentiating neurons in a dorsomedial position (DM). They show differences in the degeneration pattern. Degenerating cells were seen primarily in the VL population. A corresponding difference was found in limb bud extirpation experiments; a massive degeneration occurred in VL cells, whereas the DM cells seemed to undergo only a severe atrophy.

The re-investigation of this phenomenon on a larger scale and extending over a longer period of development necessitates a substantial revision and provides supplementation of these data. Most importantly, the extension of the observations beyond day 8 has revealed a second period of degeneration, affecting the DM neuron population in both brachial and thoracic ganglia. Carr and Simpson (1978) have reported similar observations (see "Discussion"). The new, more detailed data have served as the base line for the study of NGF effects on degeneration.

\section{Materials and Methods}

Two groups of chick embryos (white Leghorn, Spafass) were used in the present study. In the first group, normal embryos were sacrificed and staged according to the Hamburger and Hamilton stage series (Hamburger and Hamilton, 1951). In a second group, the embryos received daily injections of nerve growth factor (NGF) after which they were treated identically to those in the normal series. In each group, five to eight embryos were sacrificed at each stage between stage 24 and stage 38 . All embryos were kept in a forced draft humidifier at $37^{\circ} \mathrm{C}$ and $70 \%$ relative humidity.

$N G F$ injections. The shells of embryos to receive NGF injections were opened on the 2nd or 3rd day of incubation using a rotary sander. Daily NGF injections were made at approximately the same time each day through this window which was sealed with a sterile coverslip and paraffin after each injection. The NGF injection was made with a 10- $\mu$ l Hamilton syringe into the yolk sacs of the embryos beginning at about stage 21. A single dose of NGF consisted of $6 \mu \mathrm{g}$ of purified NGF in 6 to $12 \mu \mathrm{l}$ of $0.9 \%$ sterile saline to which a solution of Millipore-filtered bovine serum albumen (BSA) had been added to produce a concentration of $6 \mathrm{mg} / \mathrm{ml}$ of BSA.

Histological preparation. The embryos were fixed by immersion in Carnoy's fluid, embedded in paraffin, and sectioned coronally at $10 \mu \mathrm{m}$. Serial sections were mounted and stained with thionine or Pappenheim's solution (methyl green and pyronin Y).

Reconstructions. To identify the individual ganglia used in this investigation, a reconstruction of the lower cervical, brachial, and upper thoracic ganglia was made for each embryo. The reconstruction was made on graph paper by marking the lengths of ganglia 12 through 18 and their relative positions along the rostrocaudal axis. The lengths and positions were drawn to scale by equating each section with a gradation along the length of the reconstruction. To ascertain the identity of the ganglia, the brachial plexus and the emergence of the main brachial nerves were reconstructed.

Method of studying degeneration. The major difficulty which one encounters in morphometric studies of embryonic spinal ganglia is the overlap of proliferation and cell death. Hughes (1961) and Prestige (1967), who have studied this phenomenon in anuran embryos, have referred to it as "turnover." It results in a continuous change in population size, since birth rates and death rates have different (and possibly unrelated) time courses. In a study of neuron degeneration in the spinal ganglia of chick embryos, Carr and Simpson (1978) computed a degeneration index which is the percentage of degenerating cells in the total population. We have not adopted this method for two reasons. First, the total population is heterogeneous; it consists of a mixture of proliferating neuronal and glial precursors as well as neurons and glia in various stages of differentiation. In addition, the ratios of these cell types are changing continuously. Second, the indices are very low (mostly between 2 and $4 \%$ ). As a result, the curves (their Fig. 5) are flat over long periods, and a visualization and evaluation of the differences between normal and NGF-treated neuron populations would have been very difficult.

In the present study, we have obtained satisfactory results by simply counting all frankly degenerating cells in every other section through a ganglion. These counts were made at every stage from stage $25\left(4^{1 / 2} \mathrm{~d}\right)$ to stage $38(12 \mathrm{~d})$. To make the project manageable, two representative ganglia were chosen: Ganglion 15 represents limb-innervating ganglia and ganglion 18 represents nonlimb ganglia. The latter is the most rostral ganglion that innervates exclusively thoracic structures since ganglion 17 contributes frequently to the brachial plexus.

Identification and counting of degenerating cells. At the magnification used $(\times 750)$, the identification of frankly degenerating cells is unequivocal. They can be distinguished clearly from mitotic cells with clumped chromatin. Degenerating cells appear in a variety of formus, ranging from multiple basophilic small spheres in an otherwise intact nucleus and perikaryon to unrecognizable fragments. Frequently, the cell mass is condensed in a deeply stained homogeneous sphere. In other instances, the cells become vacuolated or ballooned. Macrophages containing debris of several cells were encountered infrequently and were counted as two degenerating cells. Further details of degeneration in sensory ganglia are dealt with extensively by Pannese (1976) who also discussed the pertinent literature. Obviously, we miss the incipient stages of degeneration which are revealed only by the electron microscope, but this is not a problem in the present study since the absolute figures are used only for comparison.

Cell counts were made separately for the VL and DM populations. Consequently, a major concern was the distinction between degenerating VL and DM cells. The two populations are not always separated by a clear demarcation line. Nevertheless, it was usually possible to identify a degenerating cell as VL or DM on the basis of its topographic position (ventrolateral or dorsomedial). In case of doubt, the size of immediately adjacent cells was used as a criterion, the VL cells being considerably 


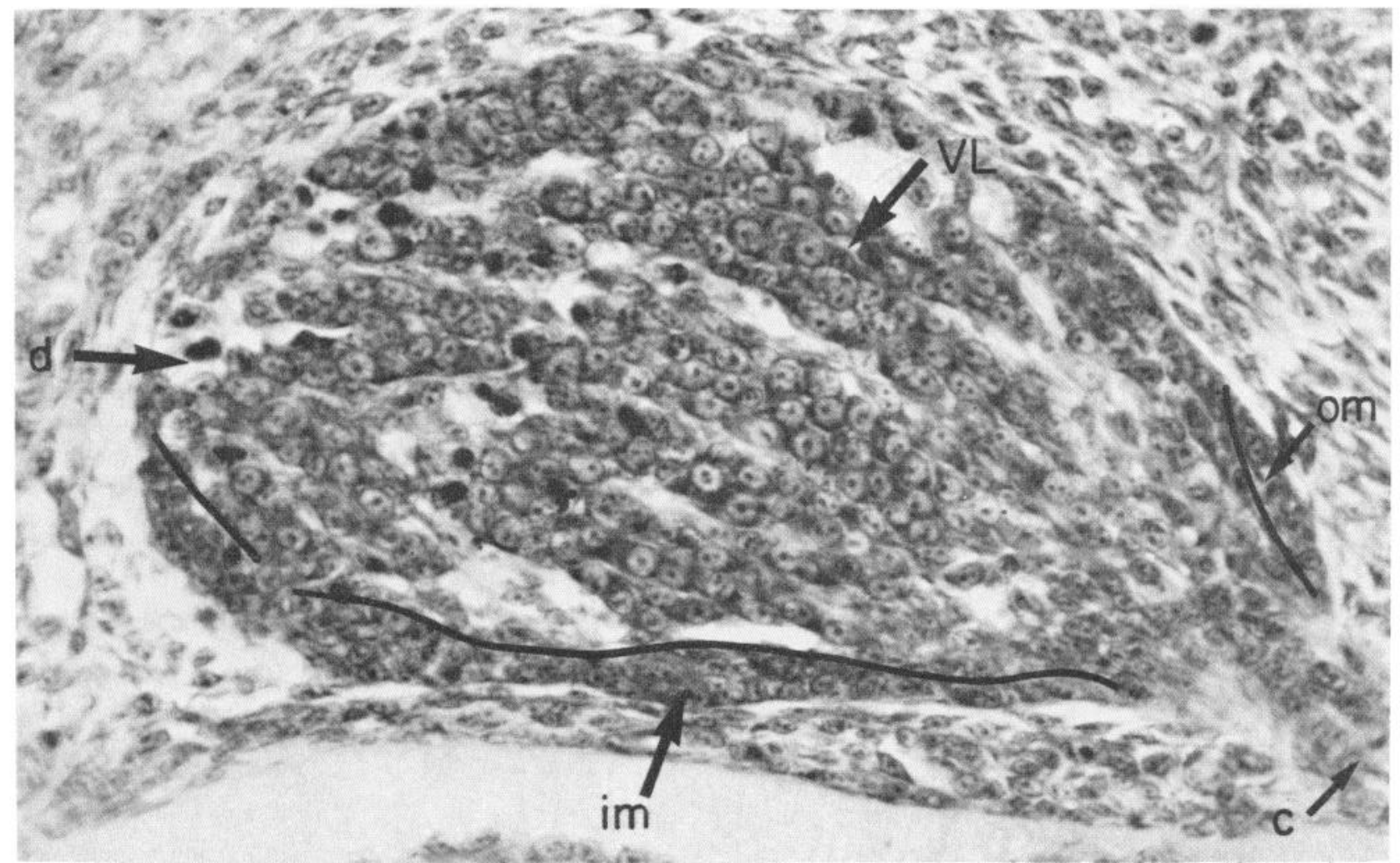

Figure $1 .^{4}$ Right thoracic ganglion 18 , stage 27 (5 to $5^{1 / 2} \mathrm{~d}$ ), at the peak of the VL degeneration period. Magnification, $\times 600$.

larger than the DM cells during the period of our study. In later stages, one has to reckon with degeneration of glia cells. In fact, occasionally, we have encountered very small, solid spheres which might be degenerating glia. They were not counted.

Both left and right ganglia 15 and 18 were counted in each embryo. Every other section was counted under oil immersion $(\times 750)$ and the totals were multiplied by 2 . For each stage, 10 to 16 ganglia were counted both in the normal and in the NGF-treated series. Most counts were made by Viktor Hamburger and some were made by $\mathrm{J}$. Brunso-Bechtold.

\section{Results \\ Histogenesis}

In the following, we describe some light microscope observations of the differentiation of spinal ganglia which are relevant to the study of neuronal death. We have made no measurements of volume increases.

\section{Thoracic ganglion 18}

Stage 24. The ganglion is composed of tightly packed cells with large nuclei surrounded by a sparse layer of cytoplasm. Most nuclei have two nucleoli. The size of the nuclei varies greatly; the largest are in the center of the ganglion and the smallest are located at the medial border. Among the latter are nuclei with dispersed chro-

\footnotetext{
${ }^{4}$ The abbreviations used on the figures are: $c$, connection with spinal cord; d, degenerating VL neuron; DM, dorsomedial population; $g$, dorsal root ganglion; im, inner marginal zone; $\mathrm{m}$, mitosis; $\mathrm{ms}$, mesonephros; om, outer marginal zone; sc, spinal cord; sm, axon bundle to mesonephros; sy, sympathetic chain ganglion; VL, ventrolateral population.
}

matin and no nucleoli which are considered to be undifferentiated cells. The larger are VL neurons in early phases of differentiation (Pannese, 1974). In this and subsequent stages, mitoses are scattered throughout the ganglion.

Stage 25. A new structure which was not clearly discernible in the preceding stage makes its appearance. A slender rim of very small, densely packed cells is found along the entire medial border of the ganglion and extending beyond the dorsal edge of the ganglion a short distance along the dorsolateral border. We have designated this zone as the inner and outer marginal zone. Some nuclei in this zone have dispersed chromatin, and others have nucleoli. This structure persists to approximately stage 31 , and as the stage-by-stage tracing shows, gives rise to the DM population. The marginal zone is set off more or less distinctly from the less densely organized population of larger VL cells. The largest VL cells are still in the center.

Stage 26. The bulk of the ganglion is composed of VL cells of different sizes. Medium-sized VL cells are adjacent to the marginal zone which is now more distinct and extends along the entire lateral and dorsomedial border. The ganglion is connected with the dorsal spinal cord by strands of large cells. At the inner border of the inner marginal zone, one finds a few very small cells intermingled with the VL cells; they represent the first definable DM cells. An increasing number of mitoses is found in the marginal zone.

Stage 27 (Figs. 1 and 2). The VL cell population becomes less densely packed. Dorsal root fibers separate VL cell groups and irregular strands of VL cells extend parallel to the fibers toward the dorsal root, but in the 
ventrolateral region, the cells have an irregular distribution. The volume of the cytoplasm is enlarged; the nuclei are eccentric. Both inner and outer marginal zones are distinct from the VL population. The inner zone is at some points interrupted by VL cells. The number of small DM cells along its central border has increased, but a distinct DM region is not yet discernible. Degenerating VL cells are scattered throughout the ganglion (Fig. 2).

Stage 28. At this stage, the number of small DM cells set apart from the marginal zone has increased and they form now a distinct group between the larger VL cells and the smaller, more densely packed marginal zone. The VL cells of varying size still occupy the bulk of the ganglion. Their loose arrangement is in contrast to the more dense packing of the DM cells. Most mitoses are found now in the inner and outer marginal zone. Counts of two ganglia showed that $78 \%$ of the mitoses are in this region. This fact and the demonstration that all small cells which later become the DM population are found exclusively along the inner border of the marginal zone identify the latter as the source of the DM population. However, it will take special labeling techniques to decide whether the newly born DM cells migrate inward or the border of the ganglion expands outward.

Stage 29 . The VL cells are spaced widely apart; groups are separated by fiber bundles. The DM population now occupies approximately the dorsal one-third of the ganglion. The demarcation line between VL and DM neurons is distinct, due to pronounced size differences and the dense packing of the DM cells. It is an irregular line, and strands of VL cells extend across the DM population toward the dorsal root. This feature is retained throughout the subsequent stages.

Stage 30. The DM population has extended further and now occupies nearly one-half of the volume of the ganglion.

Stage 32. According to Carr and Simpson (1978), all DM cells have withdrawn from the mitotic cycle at $7 \frac{1}{2} \mathrm{~d}$. The marginal zone has disappeared, except for small remnants. The topographic relation of VL and DM cells is definitely established. The largest DM cells are near the demarcation line and the smallest at the medial border of the ganglion. A new feature has appeared: A narrow row of large VL cells extends along the lateral border of the ganglion almost to the point of emergence of the dorsal root.

Stage 35 (Fig. 3). The 81/2- to 9-d ganglion represents the frequently described organization of the ganglion with clearly defined VL and DM populations. The demarcation line between them is always irregular. Strands of large VL cells extend toward the dorsal root, cutting across the DM population. Dorsal root fiber bundles separate DM cells which are aligned in irregular longitudinal rows in the dorsal part of the ganglion. Considerable size differences are found among both VL and DM populations. The smallest DM cells are still found at the medial border. Scattered degenerating cells are found almost exclusively within the DM population.

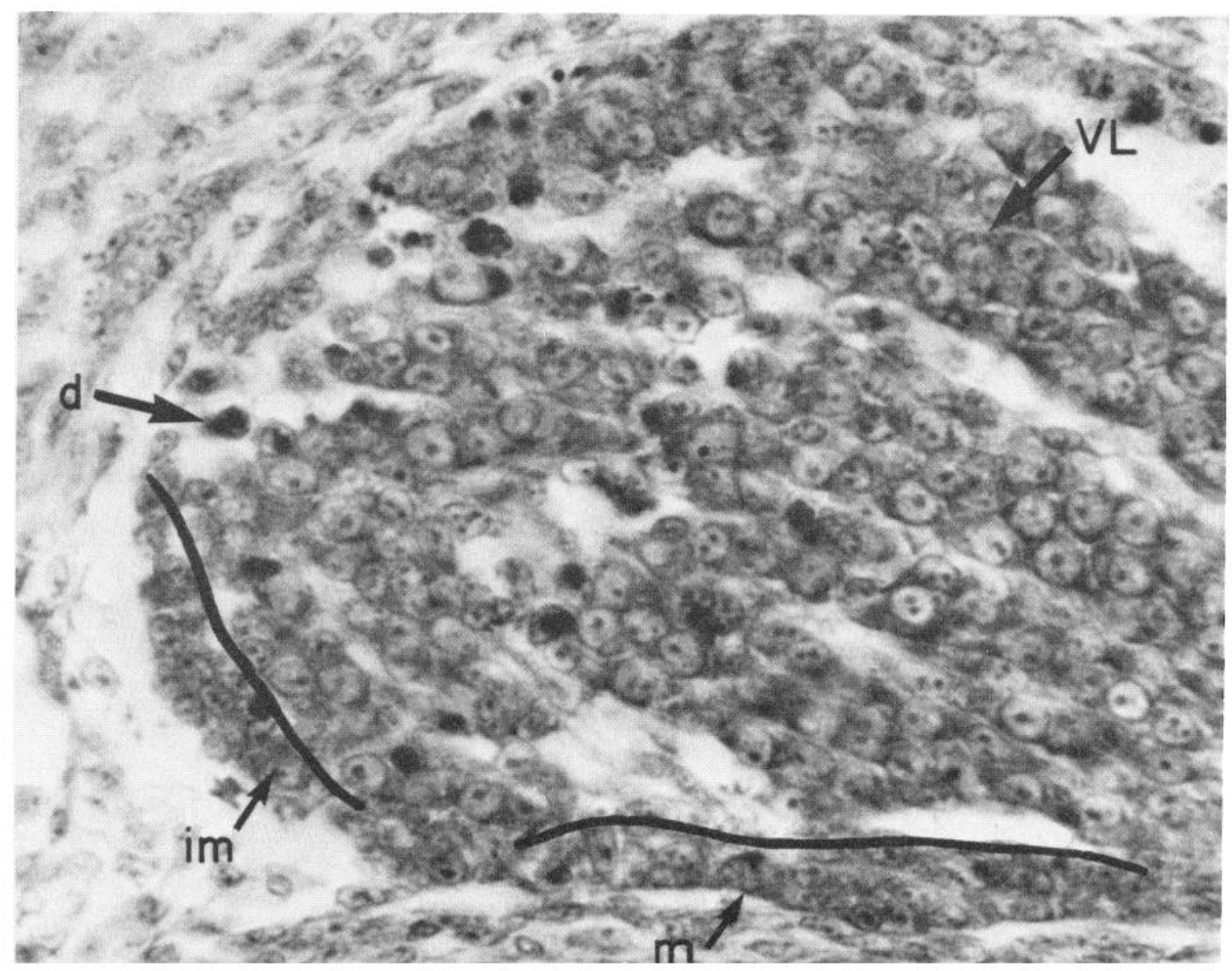

Figure 2. Enlargement of ventral part of the same ganglion as in Figure 1. Magnification, $\times 1000$. 


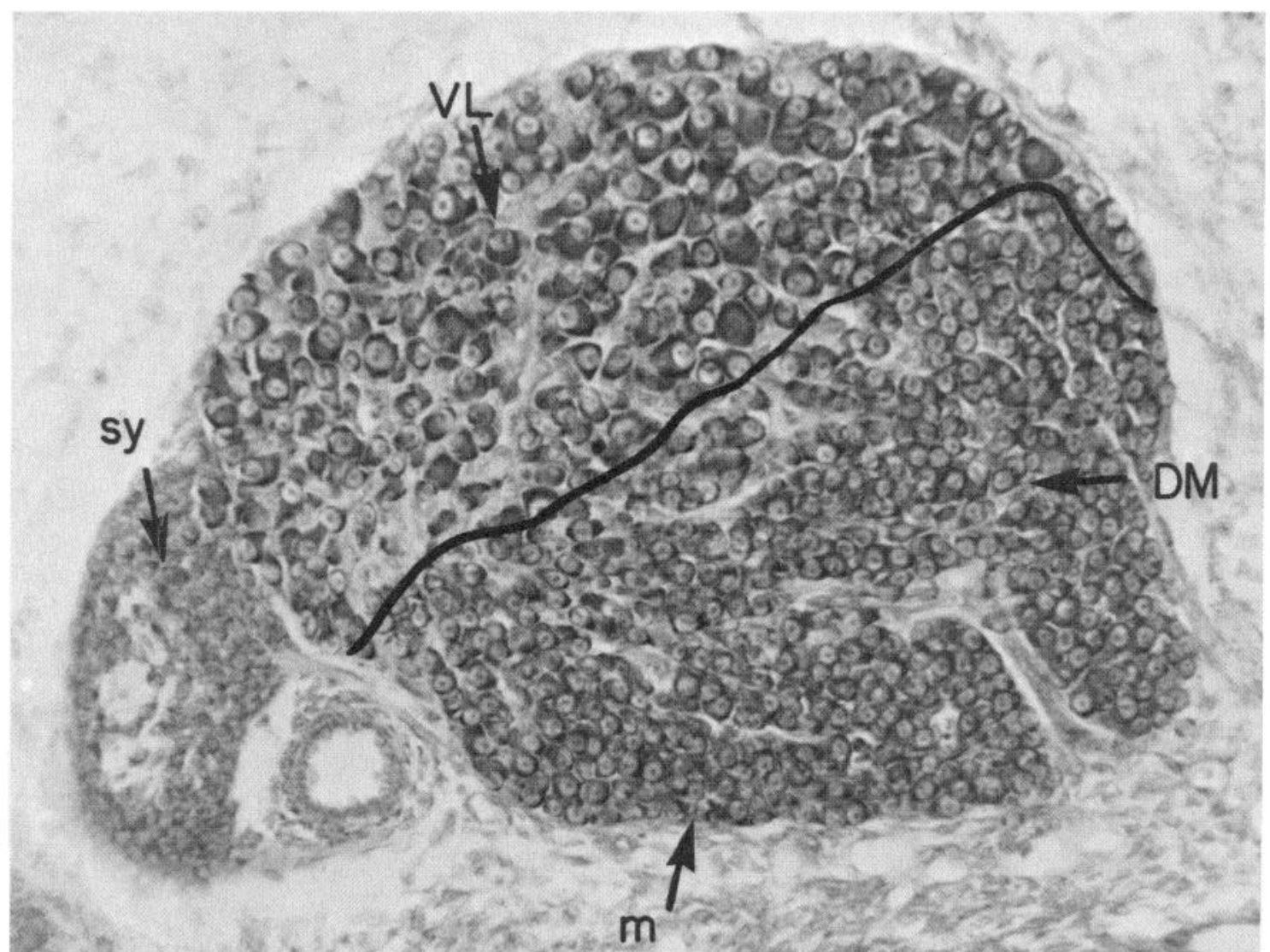

Figure 3. Right thoracic ganglion 18 , stage 35 ( $81 \frac{1}{2}$ to 9 d). The fusion of the two ganglia is atypical. Magnification, $\times 350$.

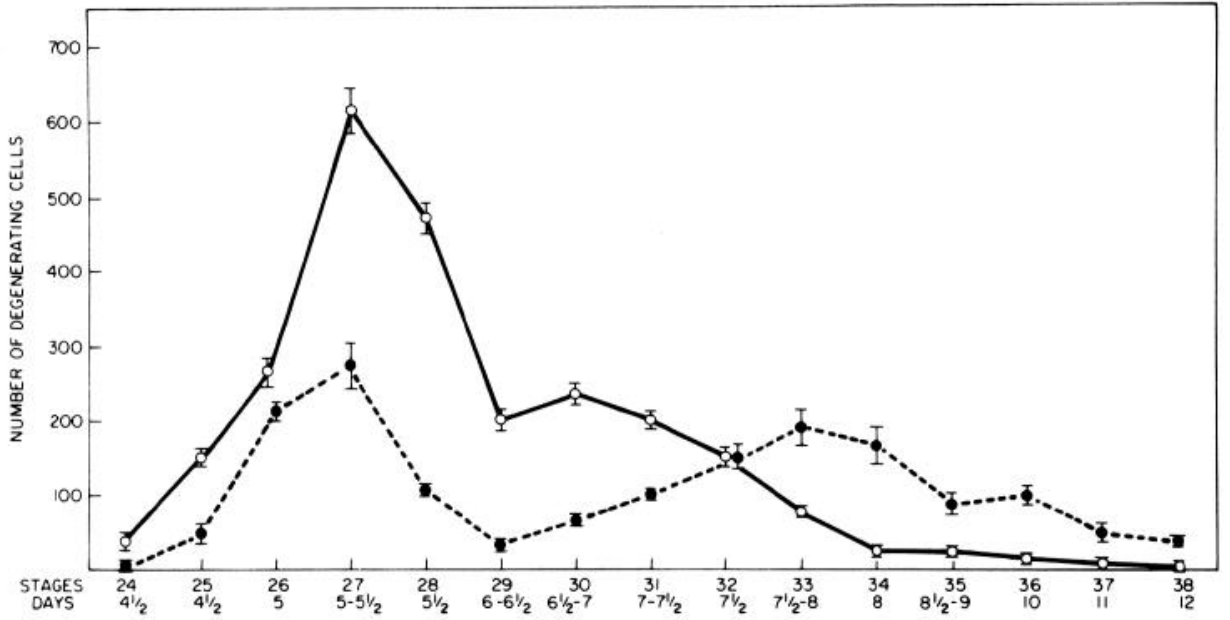

Figure $44^{5}$ Degeneration in the VL population of thoracic ganglion 18.

It should be mentioned that from stage 24 on, we have observed a small number of very large cells which stand out among the rest of the VL cells.

The differentiation of brachial ganglion 15 does not differ from that of thoracic ganglion 18 except in one small detail. No bands of VL cells were found to extend

\footnotetext{
${ }^{5}$ Figures 4 to 7 show the numbers of degenerating neurons with the standard error of the mean. Each point represents the average of 10 to 16 ganglia. The symbols used are: $\mathrm{O}_{-} \mathrm{O}$, normal; - treated.
}

across the DM population at any stage. The demarcation line always separates two homogeneous populations.

\section{Degeneration in Normal Development}

\section{Thoracic ganglion 18}

In the VL population (Fig. 4), the number of degenerating cells rises sharply beginning at stage $25\left(4^{1 / 2} \mathrm{~d}\right)$; it reaches a peak at stage 27 and then declines immediately. The steep downward slope is broken at stage $29(6 \mathrm{~d})$ and degeneration continues at a slower pace to reach a 


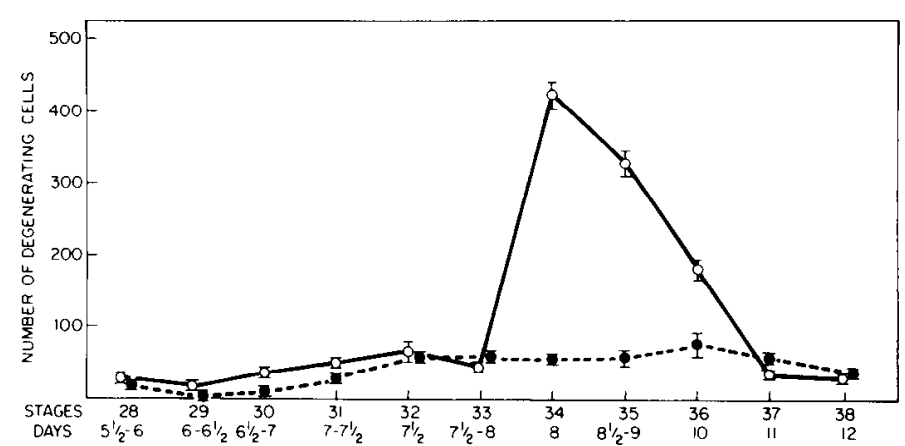

Figure 5. Degeneration in the DM population of thoracic ganglion 18.

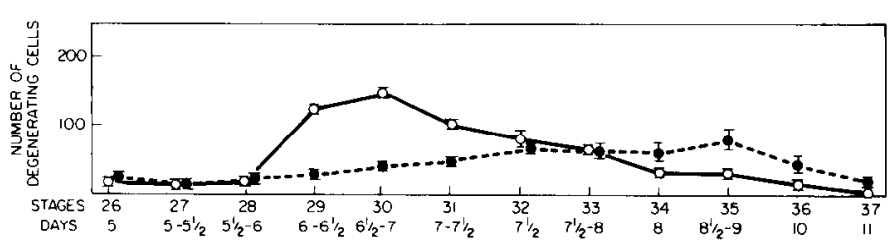

Figure 6. Degeneration in the VL population of brachial ganglion 15.

base level (see "Discussion" for definition of this term) at stage $34(8 \mathrm{~d})$. The period of massive cell loss between stages 24 and 29 lasts only $2 \mathrm{~d}$. According to the autoradiographic study of Carr and Simpson (1978), proliferation in the VL population ceases at $6^{1 / 2} \mathrm{~d}$. Hence, during the steep phase of degeneration, the heavy loss of cells is compensated for, in part, by the addition of new neurons, but this does not hold for the second phase with the less prominent slope.

In the DM population (Fig. 5), a steep rise in the number of degenerating cells begins at stage 33 ; thus, the degeneration periods for the VL and DM cells barely overlap. The peak of DM degeneration is reached within half a day, at stage 34 , and a sharp decline begins at stage 35 . The base line is reached at stage 37 , hence the duration of the degeneration process is 3 to $3^{1 / 2} \mathrm{~d}$. Proliferation of DM cells terminates at 7/1/2 $\mathrm{d}$ (Carr and Simpson, 1978). Therefore, there is no "turnover" in the DM population, and it will be possible to obtain precise figures for the actual cell loss by counts at the beginning and end of the degeneration period.

\section{Brachial ganglion 15}

It has been reported in 1949 (Hamburger and LeviMontalcini, 1949) that degeneration in the brachial ganglia is either much lower than in non-limb ganglia or entirely absent. In view of the new data, a correction in this statement is necessary. There is a distinct degeneration period in the VL population which begins at stage 27 and ends at stage 34; however, the peak at stage 30 is much lower than the VL peak in ganglion 18, amounting to less than $25 \%$ of the latter (Fig. 6). We have not found a ganglion in which degeneration is completely absent. Another difference is noteworthy; the degeneration periods of VL cells in ganglia 15 and 18 are not synchronous; that of ganglion 15 is delayed by approximately $1 \frac{1 / 2}{d}$, beginning at stage 27 , when degeneration in ganglion 18 is already at its peak.
The same holds for the degeneration in the DM population (Fig. 7). In both ganglia, degeneration begins after stage 33 . However, in ganglion 18 , the peak is reached at stage $34(8 \mathrm{~d})$, whereas in ganglion 15 , the peak is reached at stage $35\left(8^{1 / 2}\right.$ to $\left.9 \mathrm{~d}\right)$; the base level is reached at stage $37(11 \mathrm{~d})$ and $38(12 \mathrm{~d})$, respectively. In other respects (including the number of degenerating cells at the peak), the curves for ganglia 15 and 18 are very similar.

\section{Degeneration in NGF-treated Ganglia}

\section{Thoracic ganglion 18}

Figure 4 gives convincing evidence for a conspicuous reduction in normally occurring neuronal death in the VL population by NGF treatment. The absolute figures are significantly lower than in untreated ganglia for stages 25 to $31\left(4^{1 / 2}\right.$ to $\left.7 \frac{1}{2} \mathrm{~d}\right)$, that is, throughout the major part of the degeneration period. At the peak of cell death at stage 27, the absolute figures for the numbers of degenerating cells in the experimental ganglia are only $40 \%$ of those for normal ganglia.

An unexpected phenomenon occurs in the second half of the observation period. Beginning at stage 29 when the absolute figures in normal ganglia have reached already a low level, the figures for the experimental ganglia begin to rise again, while those in normal ganglia continue to drop. At stage 32 , the curves diverge, and the figures for NGF-treated ganglia reach a second peak at stage $33\left(7 \frac{1}{2}\right.$ to $\left.8 \mathrm{~d}\right)$ when cell death in normal ganglia approaches the base line. The decline to the base line in the experimental ganglia is delayed by several days compared to normal ganglia. An explanation of this puzzling phenomenon is offered under "Discussion."

The role of NGF as a trophic maintenance factor is demonstrated most convincingly in the response of the DM cells. The doses which we have administered were sufficient to save the lives of practically all DM cells. We observed again a slight rise in the number of degenerating cells toward the end of the normal degeneration period, corresponding to that seen in the VL population.

\section{Brachial ganglion 15}

A comparison of Figures 6 and 7 shows that the effect of NGF on both populations is similar to that on the DM population in ganglion 18. In other words, NGF treatment reduces cell loss almost to the base level. However, we encounter in both populations the same phenomenon

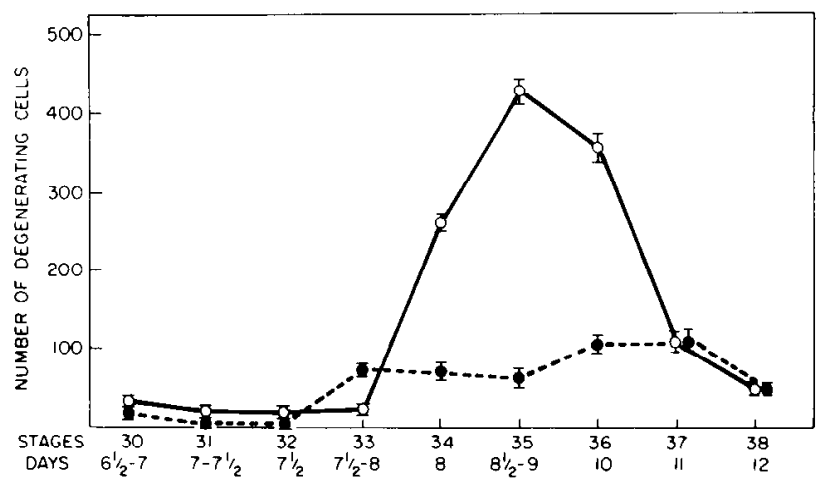

Figure 7. Degeneration in the DM population of brachial ganglion 15. 
described above, namely a slight rise in the number of degenerating cells during the terminal phase of the degenerating period. Here one cannot speak of a second peak, because there is a plateau during the main phase of the normal degeneration period. However, it is interesting to note that, as in ganglion 18 , the belated peak in the VL population occurs very late, after the normal degeneration process has ended, whereas in the DM population, the belated peak is found before the normal degeneration process is terminated.

\section{Discussion}

\section{Sporadic neuronal death: Definition of "base line"}

It is well known that scattered degenerating cells are present in many, if not all, embryonic structures. We shall refer to this phenomenon as "sporadic death." It is clearly distinguished from "phylogenetic" death, that is, the degeneration of rudimentary organs such as pro- and mesonephros, aortic arches in higher vertebrates, the tail in metamorphosing anurans, and other instances of patterned degeneration (see Ernst, 1926; Glücksmann, 1951; Saunders, 1966). The developing nervous system is no exception. However, sporadic death is rarely mentioned because it is overshadowed by a third type of degeneration characteristic of the nervous system only, the socalled natural or normal neuronal death caused by restrictive conditions at the target area of the axons. Yet, sporadic neuronal death cannot be ignored though it may play only a minor role. Figures 4 to 7 show that a small number of degenerating cells are found in all stages preceding and following the periods of natural neuronal degeneration in VL and DM populations. (VL refers to early differentiating, large neurons in a ventrolateral position and DM to late differentiating, small neurons in a dorsomedial position.) Sporadic death involves usually 50 cells or less, per ganglion. This figure is very low, indeed, if one considers that from 5 or $6 \mathrm{~d}$ onward, the total population of a ganglion amounts to several thousand neurons. We have set arbitrarily a figure of 50 or less as the base line for neuronal death. Considering sporadic death, the terms "normal," "natural," or "physiological neuronal death" applied to patterned neuronal death are ambiguous. The term "target-related neuronal death" would be more precise. We have suggested elsewhere the term "neurothanasia" (Hollyday and Hamburger, 1976).

Sporadic death could be caused by genetically determined deficiencies in neurons or their precursors or by extrinsic conditions such as inadequate access to metabolites. We can confirm the observation of Pannese (1976) that occasionally, undifferentiated neuron precursor cells are found in a pyknotic condition. We have found also pairs of degenerating cells which lie close together and seem to be daughter cells of an immediately preceding cell division. Perhaps, their death is due to an abnormality in the mitotic process.

In summary, it is true that the death of some neurons is caused by factors other than restrictive conditions at the target area of their axons. It should be added that we have paid attention to the distribution of these cells. They seem to be randomly scattered in the population, but a more detailed analysis may reveal a pattern.

\section{Some caveats concerning the interpretation of the data}

The rationale for presenting the data in terms of absolute numbers of degenerating cells without reference to total populations is given under "Materials and Methods." This procedure involves some pitfalls and the data as presented in Figures 4 to 7 should not be overinterpreted. For instance, on the abscissa, the stages are plotted at equal distances from each other. One should be aware that up to stage 34 , this scale is entirely unrelated to the chronological time scale. From stage 34 to hatching, the stages are $1 \mathrm{~d}$ apart. Our choice of stages as units seems justified in that it gives a clear picture of the beginning, peak, and end of the degeneration periods. Furthermore, the curves are well suited for comparison of the degeneration process between VL and DM populations and between normal and NGF-treated ganglia.

Similar reservations hold for the ordinates. The absolute figures have little significance by themselves because they have no relation to the total populations, which are constantly changing. But they permit a clear assessment of NGF effects.

\section{Neuronal death in normal spinal ganglia}

The distribution patterns and other aspects of neuron degeneration as described first by Hamburger and LeviMontalcini (1949) were outlined briefly in the introduction. Now we can give a much more precise account on the basis of better quantification (counts as against estimates), the use of the stage series, and the extension of the study to $12 \mathrm{~d}$ of incubation.

Thoracic ganglion 18 (Figs. 4 and 5). The description of the essential features of degeneration in the VL population given in 1949 was found to be correct: Degeneration begins at $4^{1 / 2} \mathrm{~d}$; it reaches a peak at 5 to $6 \mathrm{~d}$ and ends after day 7 . The present data show that the terminal phase actually extends to day 8 . The break in the downward slope of the curve at stage 29 in ganglion $18 \mathrm{had}$ not been observed before; a possible explanation for this is discussed below.

The most important new finding is the establishment of a distinct degeneration period for the DM population which has characteristics similar to that of the VL population. The two periods are clearly separate with only a slight overlap at stages 33 and 34 . The degeneration in the DM period begins at stage 33 ; it shows a steep rise to a peak at stage 34 and a sharp decline to the base level which is reached at stage 37 . There is no break in the downward slope, comparable to that found in the VL curve. The highest counts in the DM population amount to only two-thirds of those in the VL population.

Brachial ganglion 15. Contrary to the claim made in 1949 that brachial ganglia show little or no degeneration, we have now established clearly defined degeneration periods for both VL and DM populations. However, it is clear from a comparison of Figures 4 and 6 that the total loss of VL cells in ganglion 15 is only a fraction of that found in ganglion 18. Perhaps the difference can be explained by the assumption that the rapidly growing limb tissues produce a larger amount of a hypothetical trophic maintenance factor than flank tissues. Another difference between ganglia 15 and 18 has been mentioned already; it concerns the time table of events. The VL 
degeneration in ganglion 15 occurs later than in ganglion 18. It begins at stage 27 when degeneration in ganglion 18 is already at its peak, and the two peaks are $1 \frac{1}{2}$ to 2 $\mathrm{d}$ apart. This peculiar feature can perhaps be explained by postulating that the steep phase of the VL degeneration curve in ganglion 18 is missing in ganglion 15 . On the other hand, the second phase in the curve for ganglion 18 which is characterized by gentle slopes shows a remarkable resemblance to the entire curve of ganglion 15 . In fact, if one extrapolates the second phase in the curve of ganglion 18 to the left, then the low curves for 15 and 18 can be practically superimposed. This similarity suggests that we may be dealing in ganglion 18 with two subpopulations with overlapping degeneration periods and that in ganglion 15, only the second subpopulation is represented. If so, then the break in the downward slope of the curve of ganglion 18 would assume a significant meaning.

Two other studies deal with degeneration in spinal ganglia quantitatively: those of Palladini (1961) and of Carr and Simpson (1978). Both have calculated degeneration indices which represent the percentage of degenerating cells in the total population. Obviously, these data cannot be related directly to our figures. Furthermore Palladini did not distinguish between the VL and DM populations and the study of Carr and Simpson ended at $9 \frac{1}{2}$ d so that they obtained an incomplete picture of degeneration in the DM population. However, both studies anticipate our finding that degeneration does occur in limb-innervating ganglia, and Carr and Simpson have described the beginning phase of the DM degeneration period in both brachial and non-brachial ganglia.

\section{Relation of degeneration to conditions at the periphery}

It is generally agreed that the massive degeneration of neurons with which we are concerned here is related to restrictive conditions prevailing at the target area of their axons and that they are subjected to a competition in which only $50 \%$ or less survive. The present model of competition is derived largely from studies of neuromuscular systems. As a result, competition is often envisaged in terms of competition for synaptic sites. Yet, degeneration in the spinal ganglia is as severe as in the motor system, although they lack the equivalent of synapses. Hence, studies like the present one can serve the purpose of restoring a broader, more balanced perspective to the problem of neurothanasia.

An important point emerges from our re-investigation of the dorsal root ganglia: The VL and DM populations have always been considered as two distinct units on the basis of their topographic separation and differences in size and in time of differentiation. We have shown now that they also have separate, clearly definable, degeneration periods, both in thoracic and brachial ganglia. One is tempted to draw the conclusion that we are dealing with two functionally homogeneous units, such as exteroand proprioceptive groups. Yet this supposition does not seem to be tenable. It was suspected from the beginning that each population might be heterogeneous, despite outward appearances (Hamburger and Levi-Montalcini, 1949, p. 463). The mapping experiments of Honig (1977;
M. G. Honig, personal communication) have given results which complicate the situation even further. She has found that HRP injections either into the skin or into hindlimb muscles of 10- to 12-d embryos label, in both instances, neurons in the VL population as well as in the DM population, implying that both populations include extero- and proprioceptive neurons. This finding is difficult to reconcile with the observation that the VL population sends out axons and is subject to degeneration several days earlier than the DM population. It will be necessary to obtain much more information concerning the projection of particular neurons to particular target sites and about the time of arrival of the axons at their respective destinations before we can give a satisfactory explanation of the different degeneration patterns.

However, it is possible at this time, to make some inferences concerning neurothanasia in the VL population of thoracic ganglia, which occurs between $4 \frac{1}{2}$ and 7 d. We should consider first the peripheral distribution of VL fibers. Silver-impregnated preparations show that at stage $25\left(4 \frac{1}{2} \mathrm{~d}\right)$, mixed nerves are formed and sensory bundles approach the skin and begin to spread under the epidermis (Fig. 8; see also Visintini and Levi-Montalcini, 1939). Hence, the majority of the early differentiating VL

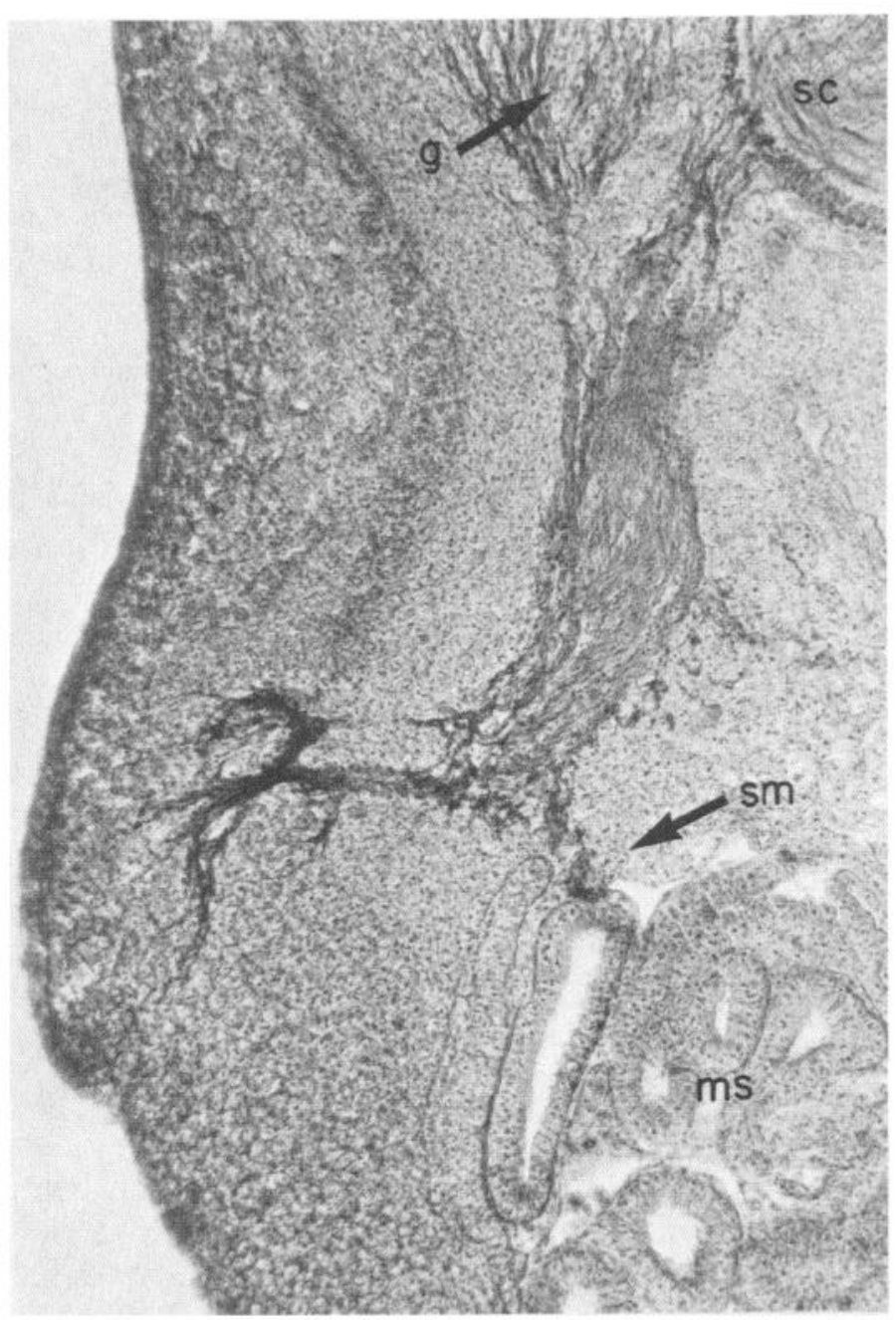

Figure 8. Peripheral distribution of sensory axons of a thoracic ganglion at stage $25\left(4^{1 / 2} \mathrm{~d}\right)$. De Castro modification of Cajal's silver impregnation. Magnification, $\times 300$. 
neurons are exteroceptive, with free nerve endings. LeviMontalcini (1964) has discovered an additional component. She observed that in $4 \frac{1}{1} 2$-d embryos, each thoracic spinal ganglion sends several strong fiber bundles to the adjacent mesonephros (Fig. 8). These are transient nerves which disappear again in subsequent days. While the significance of this curious phenomenon remains obscure, the regression of the aberrant nerves undoubtedly contributes to the high figures of pyknotic cells which one finds in 5- and 6-d thoracic ganglia.

We were impressed by the very early beginning of degeneration. As shown in Figure 4, degeneration in the VL population of ganglion 18 is well above the base line at stage $25\left(4^{1 / 2} \mathrm{~d}\right)$ and rises rapidly during the following day. Pannese (1976), in his electron microscopic study of degeneration in chick spinal ganglia, found that the cells which die between 4 and $7 \mathrm{~d}$ (and can therefore be identified as VL neurons) are "primitive neuroblasts" which are defined as bipolar neuroblasts, or transitional stages between "primitive" and "intermediate neuroblasts," the latter representing bipolar neurons in which the two processes approach each other but have not yet reached the stage of pseudo-unipolar neurons. Clearly, these sensory neurons become sensitive to trophic deficiencies at a much earlier stage of differentiation than, for instance, the neurons of the lateral motor column or the ciliary ganglion.

The immaturity of the ganglion during the early phase and during the peak of degeneration is reflected also in the fact that proliferation proceeds simultaneously with axon outgrowth and degeneration. Carr and Simpson (1978) have found that proliferation in the VL population of cervical and brachial ganglia continues to $6^{1 / 2} \mathrm{~d}$ (approximately stages 29 to 30 ) and we assume that proliferation in thoracic ganglia is terminated at approximately the same time. This means that we are dealing with "turnover," that is, simultaneous birth and death of neurons in ganglion 18, between stages 25 and 30.

On the basis of these findings, we shall make an attempt to understand the factors which lead to degeneration in spinal ganglia. Regression begins shortly after some fiber bundles have reached the dermis or mesonephros, while the growth cones of other fibers traverse the mesenchyme. There can hardly be a competition of free endings for territory, because, according to our observations on silver preparations, they have not spread extensively even at the time when degeneration is at its peak, and furthermore, because particularly in the early phase of degeneration, only a fraction of the fibers which the ganglion will produce are competing with each other.

For competition to occur under these circumstances, we envisage the interplay of several variables, on a microscale. These include $(i)$ the limited production of a hypothetical trophic agent, or its limited accessibility to the embryonic sensory neurons; (ii) high susceptibility of the neurons to deficiencies in the trophic agent; and (iii) local differences in the density of nerves. Pilar et al. (1980), in their analysis of competition in the ciliary nerve, have called attention to the third point and found it of some importance in their system. We shall comment briefly on the second point. There is good evidence for somatic motor systems and for the ciliary ganglion that all neurons have the same potential for survival and that their fate is decided at the target area. The best evidence comes from experiments in which neurons which would normally die are rescued by enlargement of the target area (Hollyday and Hamburger, 1976; Narayanan and Narayanan, 1978), by reduction of the number of competing neurons (Pilar et al., 1980), or by treating chick embryos with neuromuscular blocking agents (Pittman and Oppenheim, 1979). This means that programmed cell death is ruled out. There is some evidence that the situation may be similar in amphibian spinal ganglia (Bibb, 1977). However, the possibilities that not all sensory neurons are "born equal" and that there exist slight differences in susceptibility to extrinsic agents cannot be dismissed. In fact, Hendry (1977) contends that during neuronal degeneration in the superior cervical ganglion of newborn rats, younger neurons have a higher mortality rate than older ones, and Prestige (1967) has found the same age difference in vulnerability in Xenopus motoneurons following limb extirpation.

What could be the source of the trophic maintenance agent? The most likely candidate is the mesenchyme which the axons traverse and the subcutaneous mesenchyme in which the nerve endings spread. The hypothetical agent would then be internalized at the free endings and transported retrogradely to the perikaryon.

We should like to carry the speculations a step further. If, indeed, we are dealing with a diffusible agent to which the axons are exposed shortly after the beginning of outgrowth, and considering the very short distances between the ganglia and their periphery and their proximity to mesoderm in 5- and 6-d embryos, then one could imagine that the agent reaches the perikarya directly. Consequently some neurons may die even before they have produced axons, due to restricted trophic conditions. We have found degenerating neurons among the very small cells in the marginal zone which appear to be undifferentiated or in a very early stage of differentiation. An interesting finding of Carr (see Hughes and Carr, 1978) could be interpreted as supporting this suggestion. She injected a single dose of $\left[{ }^{3} \mathrm{H}\right]$ thymidine into $5^{1 / 2}-\mathrm{d}$ embryos (that is, at the peak of VL degeneration) and sacrificed them $20 \mathrm{hr}$ later. Label was found in $13 \%$ of the degenerating cells. This seems to imply that neuron precursors die while they are still in the process of proliferation. Most mitotic cells would be classified as undifferentiated cells; but Pannese (1974) has described dividing cells in spinal ganglia which are in transition from undifferentiated cells to bipolar neuroblasts.

It is clear that the situation during the periods of natural neuronal death in sensory ganglia and motor systems is different in some fundamental aspects which we shall summarize briefly.

In the several somatic motor systems which have been investigated and in the ciliary ganglion in which the analysis has been carried to even greater detail, it has been found that a considerable time span intervenes between the birth of neurons and the beginning of degeneration. During this period, the motor pools are assembled, they have attained their total pre-degeneration numbers, they have sent out axons, and all or most of the axons have reached their target area which becomes 
the arena for the "struggle for survival." This implies that motoneurons including the ciliary neurons can sustain themselves for a considerable period before they become dependent on the conditions prevailing at the target area. For instance, in the chick embryo, the neurons of the lateral motor column, most of which are born before stage $23(4 \mathrm{~d})$ (Hollyday and Hamburger, 1977) do not enter the degeneration phase until $2 \frac{1}{2}$ to $3 \mathrm{~d}$ later. In the trochlear nucleus, the period between the time of arrival of axons at their target at $5 \mathrm{~d}$ and the beginning of their degeneration is anywhere between 4 and $10 \mathrm{~d}$ (Cowan and Wenger, 1967). In the ciliary ganglion, the interval extends from 5 to $9 \mathrm{~d}$ (stages 25 to 35; Landmesser and Pilar, 1974b). During this period, all of the axons of the neurons under consideration have reached their target area (see Prestige and Wilson, 1972, for amphibian motor roots; Chu-Wang and Oppenheim, 1978, for motor roots in the chick embryo; Landmesser and Pilar, 1976, for chick ciliary ganglion), and the neurons are in an advanced stage of differentiation when degeneration begins. For instance, in the most thoroughly analyzed case, the ciliary ganglion, the neurons are capable of impulse transmission, and functional synapses from the preganglionic axons have formed on their perikarya, before the inception of the degeneration period (Landmesser and Pilar, 1974a, b).

By contrast, the sensory VL cells are very immature when selective cell death occurs; they cannot maintain themselves for any length of time in the absence of an extrinsic maintenance agent. Furthermore, outgrowth of some axons and regression of others occurs simultaneously; hence, at any given time, individual axons compete only with a fraction of the total number of axons emerging from a ganglion and not with all the axons belonging to a neuronal assembly, as is the case in the motor systems.

Finally, a major difference between the sensory and motor systems, is, of course, the absence in the former of peripheral synapses. Therefore, competition for synaptic sites can be ruled out as the cause of the neuronal degeneration and we have to resort to the more general notion of competition for a trophic maintenance agent.

We conclude that neither our tentative model for competition in sensory ganglia nor the present model for competition in motor systems can be generalized. It is possible that, indeed, the mechanisms which regulate neurothanasia in the two neuron types are different, though the basic postulate that both types of neurons require a trophic maintenance agent (or agents) remains valid.

\section{Effects of NGF on degeneration}

We have emphasized that the amount of trophic agent produced at the time of outgrowth of axons from the spinal ganglia is critical for their survival. We know also that NGF can sustain dissociated sensory neurons in vitro (Levi-Montalcini and Angeletti, 1963; Greene, 1977). The question then arose whether NGF supplied to ganglia in vivo can supplement the naturally produced trophic agent and thus rescue neurons which would normally die. Our NGF injection experiments have answered the question in the affirmative. Daily injections of $6 \mu \mathrm{g}$ of
NGF into the yolk sac from stage $21\left(3^{1 / 2} \mathrm{~d}\right)$ until the time of sacrifice have reduced neuronal death in the VL population of ganglion 18 substantially and have rescued practically all VL cells in brachial ganglion 15 and all DM cells in both ganglia.

This findings disposes of the widely held belief that VL cells are refractory to NGF. It adds a new facet to the many known faculties of NGF. The assertion that VL cells are not responsive to NGF is based on unwarranted generalizations from limited data in several studies which by themselves are not in conflict with our data. For instance, it was pointed out that VL axons do not invade intra-embryonic sarcomas, whereas DM axons do so in large numbers. Consequently, the ensuing hyperplasia of the sensory ganglia, which is due to the tumor-produced NGF is restricted to the DM population (Levi-Montalcini and Hamburger 1951).

However, for unknown reasons, sensory and sympathetic fibers do not enter the tumor until $7 \mathrm{~d}$, that is, after the time during which, according to our present findings, NGF is effective in the VL population. The failure of VL axons to enter the tumor could be unrelated to NGF. In other experiments, NGF from snake venom or mouse salivary glands was injected into the yolk sac resulting in hyperplasia of sympathetic and sensory ganglia (Levi-Montalcini, 1958). Again, the hyperplasia was confined to the DM population. The experiments, however, were performed on 7- to 10-d embryos; they do not cover the earlier stages, for which we have documented NGF effects on the survival of VL cells. In the trigeminal ganglion in which VL and DM populations are also represented, Levi-Montalcini (1962) separated the two populations surgically in 9-d embryos and grew them separately in vitro with $\mathrm{NGF}$ added to the medium. The DM fragments produced a halo of fibers within $18 \mathrm{hr}$, but the VL fragments did not show fiber outgrowth. They might have done so, if the experiment had been done on younger embryos. However, the result can be interpreted in another way, namely in terms of the dissociation of two major NGF effects. One could assume that NGF as a trophic molecule maintains VL cells under adverse conditions, but that it cannot induce axon outgrowth. In another in vitro study, Weis $(1970,1971)$ exposed embryonic spinal ganglia to NGF. He came to the conclusion "that NGF maintains and stimulates only the later differentiating mesio-dorsal neuroblasts" (Weis, 1971, p. 117). Here again, an unjustified generalization is made from data on ganglia of older embryos $\left(7 \frac{1}{2}\right.$ to $8 \mathrm{~d}$ ). However, the in vitro experiments of Ebendal (1979) did extend to earlier stages. He observed fiber outgrowth in response to NGF as early as stages 26 to 27 (5 to $5 \frac{1 / 2}{d}$ ). The early response can be attributed to VL cells. If these findings are confirmed on a larger scale, then the capacity of NGF to elicit axon outgrowth from VL neurons, as well as to support their survival, would have been established.

It will be remembered that the response of VL neurons begins at remarkably early stages. Injections beginning at stage $21\left(3^{1 / 2} \mathrm{~d}\right)$ have a demonstrable life-saving effect at stage $25\left(4 \frac{1}{2} \mathrm{~d}\right)$. This is the earliest response to NGF reported so far.

Responses of $D M$ cells to NGF have been investigated 
frequently. We add to the list of effects the capacity of NGF to maintain the life of embryonic DM cells which would have normally died in the embryo.

Dosage. The finding that NGF rescues only $60 \%$ of the $V L$ neurons in ganglion 18 but practically all of the VL cells in brachial ganglion 15 and all of the DM cells in both ganglia can be attributed to a dosage effect. The number of degenerating cells in the VL population of ganglion 18 is substantially higher than that in the other populations, and higher dosages might have eliminated the death of VL cells altogether. The VL neurons of ganglion 18 are at a considerable disadvantage for another reason. At the time when degeneration reaches its peak in this population (at stage 27), the embryos had received only two NGF injections. The peak of VL degeneration in ganglion 15 occurs a day later, and furthermore, the absolute number of degenerating VL cells in ganglion 15 is considerably lower than in ganglion 18 . The complete rescue of $D M$ neurons in both ganglia can be attributed to the fact that by the time DM degeneration occurs, around day 8 , the embryo has received five injections.

Finally, we should comment on the second peak of the NGF curve in Figure 4 and on the much lower but noticeable peaks in the NGF curves of Figures 5 to 7 . It is characteristic of all of them that they occur toward the end of the normal degeneration period in the DM populations and even later, after the end of the normal degeneration period in VL populations. The most plausible explanation is again in terms of a dosage effect. The dosages which we have administered were apparently sufficient to keep most, or all, of the cells which would have normally died alive for a short period. But in later stages (during which the NGF curves rise), the amount of accumulated NGF was apparently not high enough to sustain the survival of all previously rescued cells. It is possible that if we had adjusted the daily dosage to the weight of the embryos rather than keeping it constant, we could have eliminated normally occurring death altogether.

\section{Concluding Remarks}

As stated in the introduction, it was our intent to address the question of whether NGF is the naturally produced trophic maintenance agent for embryonic sensory neurons, using the in vivo administration of NGF. We have demonstrated that NGF can rescue sensory neurons in the embryo at exactly the time when they would have died without NGF supplementation. Hence, NGF can compensate for a deficiency in the supply of the naturally produced trophic agent. These findings strengthen the notion that NGF is indeed the naturally produced trophic agent for sensory ganglia.

\section{References}

Bibb, H. D. (1977) 'The production of ganglionic hypertrophy in Rana pipiens larvae. J. Exp. Zool. 200: 265-276.

Brunso-Bechtold, J. K., and V. Hamburger (1979) Retrograde transport of nerve growth factor in chicken embryo. Proc. Natl. Acad. Sci. U. S. A. 76: 1494-1496.

Carr, V. M., and S. B. Simpson (1978) Proliferative and degenerative events in the early development of chick dorsal root ganglia. J. Comp. Neurol. 182: 727-740.
Chu-Wang, I. W., and R. W. Oppenheim (1978) Cell death of motoneurons in the chick embryo spinal cord. II. A quantitative and qualitative analysis of degeneration in the ventral root, including evidence for axon outgrowth and limb innervation prior to cell death. J. Comp. Neurol. 177: 59-86.

Cowan, W. M., and E. Wenger (1967) Cell loss in the trochlear nucleus of the chick during normal development and after radical extirpation of the optic vesicle. J. Exp. Zool. 164: 267280.

Ebendal, T. (1979) Stage-dependent stimulation of neurite outgrowth exerted by nerve growth factor and chick heart in cultured embryonic ganglia. Dev. Biol. 72: 276-290.

Frnst, M. (1926) Ueher Untergang von Zellen während der normalen Entwicklung bei Wirbeltieren. Z. Anat. Entw. Gesch. 79: 228-262.

Glücksmann, A. (1951) Cell deaths in normal vertebrate ontogeny. Biol. Rev. 26: 59-86.

Greene, L. A. (1977) Quantitative in vitro studies on the nerve growth factor (NGF) requirement of neurons. Dev. Biol. 58: 106-113.

Hamburger, V., and H. L. Hamilton (1951) A series of normal stages in the development of the chick embryo. J. Morphol. 88: 49-92.

Hamburger, V., and R. Levi-Montalcini (1949) Proliferation, differentiation and degeneration in the spinal ganglia of the chick embryo under normal and experimental conditions. J. Exp. Zool. 111: 457-502.

Hendry, I. A. (1977) Cell division in the developing sympathetic nervous system. J. Neurocytol. 6: 299-309.

Hollyday, M., and V. Hamburger (1976) Reduction of the naturally occurring motor neuron loss by enlargement of the periphery. J. Comp. Neurol. 170: 311-320.

Hollyday, M., and V. Hamburger (1977) An autoradiographic study of the formation of the lateral motor column in the chick embryo. Brain Res. 132: 197-208.

Honig, M. G. (1977) Outgrowth of cutaneous and proprioceptive neurons from chick embryo dorsal root ganglia. Soc. Neurosci. Abstr. 3: 108.

Hughes, A. F. (1961) Cell degeneration in the larval ventral horn of Xenopus laevis. J. Embryol. Exp. Morphol. 9: 269284.

Hughes, A. F., and V. M. Carr (1978) The interactions of periphery and center in the development of dorsal root ganglia. In Handbook of Sensory Physiology. Vol. 9: Development of Sensory Systems, M. Jacobson, ed., pp. 85-114, Springer Verlag, Berlin.

Landmesser, L., and G. Pilar (1974a) Synapse formation during embryogenesis on ganglion cells lacking a periphery. J. Physiol. (Lond.) 241: 715-736.

Landmesser, L., and G. Pilar (1974b) Synaptic transmission and cell death during normal ganglionic development. J. Physiol. (Lond.) 241: 737-749.

Landmesser, L., and G. Pilar (1976) Fate of ganglionic synapses and ganglion cell axons during normal and induced cell death. J. Cell Biol. 68: 357-374.

Levi-Montalcini, R. (1958) Chemical stimulation of nerve growth. In A Symposium on the Chemical Basis of Development, W. D. McElroy and B. Glass, eds., pp. 646-664, The Johns Hopkins Press, Baltimore.

Levi-Montalcini, R. (1962) Analysis of a specific nerve growth factor and of its antiserum. Sci. Repts. Ist. Super. Sanità, 2: $345-368$.

Levi-Montalcini, R. (1964) Events in the developing nervous system. Prog. Brain Res. 4: 1-29.

Levi-Montalcini, R., and P. Angeletti (1963) Essential role of the nerve growth factor in the survival and maintenance of dissociated sensory and sympathetic embryonic nerve cells in vitro. Dev. Biol. 7: 653-659.

Levi-Montalcini, R., and V. Hamburger (1951) Selective 
growth-stimulating effects of mouse sarcoma on the sensory and sympathetic nervous system of the chick embryo. J. Exp. Zool. 116: 321-362.

Narayanan, C. H., and Y. Narayanan (1978) Neuronal adjustments in developing nuclear centers of the chick embryo following transplantation of an additional optic primordium. J. Embryol. Exp. Morphol. 44: 53-70.

Palladini, G. (1961) Neurodegenerazione nella ontogenesi di Quaglio (Coturnix Coturnix japonica T.e S.) Riv. Biol. 7: 383-400.

Pannese, E. (1974) The histogenesis of the spinal ganglia. Adv. Anat. Embryol. Cell Biol. 47: 7-97.

Pannese, E. (1976) An electron microscopic study of cell degeneration in chick embryo spinal ganglia. Neuropathol. Appl. Neurobiol. 2: 247-267.

Pilar, G., L. Landmesser, and L. Burstein (1980) Competition for survival among developing ciliary ganglion cells. J. Neurophysiol. 43: 233-254.

Pittman, R., and R. W. Oppenheim (1979) Cell death of motoneurons in the chick embryo spinal cord. IV. Evidence that a functional neuromuscular interaction is involved in the regulation of naturally occurring cell death and the stabilization of synapses. J. Comp. Neurol. 187: 425-446.

Prestige, M. C. (1967) The control of cell number in the lumbar ventral horn during the development of Xenopus laevis tadpoles. J. Embryol. Exp. Morphol. 18: 359-387.

Prestige, M. C., and M. A. Wilson (1972) Loss of axons from ventral roots during development. Brain Res. 41: 467-470.

Saunders, J. W. (1966) Death in embryonic systems. Science 154: 604-612.

Visintini, F. and R. Levi-Montalcini (1939) Relazione tra differenziazione strutturale e funzionale dei centri e delle vie nervose nell'embrione di pollo. Arch. Suisses Neurol. Psychiatry 43: $1-45$.

Weis, P. (1970) The in vitro effect of the nerve growth factor in chick embryo spinal ganglia-a light microscopic evaluation. J. Embryol. Exp. Morphol. 24: 381-392.

Weis, P. (1971) The in vitro effect of the nerve growth factor in chick embryo spinal ganglia: An electron microscopic evaluation. J. Comp. Neurol. 141: 117-132. 